\title{
Towards a stand-alone high-throughput EUV actinic photomask inspection tool - RESCAN
}

\author{
Rajendran Rajeev ${ }^{\mathrm{a}}$, Iacopo Mochi ${ }^{\mathrm{a}}$, Patrick Helfenstein ${ }^{\mathrm{a}}$, Istvan Mohacsi ${ }^{\mathrm{a}}$, Sophie Redford ${ }^{\mathrm{a}}$, \\ Aldo Mozzanica ${ }^{\mathrm{a}}$, Bernd Schmitt ${ }^{\mathrm{a}}$, Shusuke Yoshitake ${ }^{\mathrm{b}}$, and Yasin Ekinci ${ }^{\mathrm{a}}$

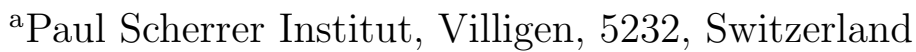 \\ ${ }^{\mathrm{b} N u F l a r e}$ Technology, Inc., Yokohoma, Japan.
}

\begin{abstract}
With extreme ultraviolet (EUV) lithography getting ready to enter high volume manufacturing, there is an imminent need to address EUV mask metrology infrastructure. Actinic defect inspection of patterned EUV photomasks has been identified as an essential step for mask qualification, but there is no commercial tool available right now. We address this gap with the RESCAN tool, a defect inspection platform being built at Paul Scherrer Institut (PSI), co-developed in collaboration with Nuflare Inc, Japan. RESCAN uses Scanning Scattering Contrast Microscopy (SSCM) and Scanning Coherent Diffraction Imaging (SCDI) for fast defect detection and fine defect localization. The development of a stand-alone tool based on these techniques relies on the availability of (1) a bright coherent EUV source with a small footprint and (2) a high frame-rate pixel detector with extended dynamic range and high quantum efficiency for EUV. We present two in-house projects at PSI addressing the development of these components: COSAMI and JUNGFRAU. COSAMI (COmpact Source for Actinic Mask Inspection), is a high-brightness EUV source optimized for EUV photons with a relatively small footprint. JUNGFRAU (adJUstiNg Gain detector FoR the Aramis User station) is a silicon-based hybrid pixel detector, developed in house at PSI and prototyped for EUV. With a high frame rate and dynamic range at 13.5 $\mathrm{nm}$, this sensor solution is an ideal candidate for the RESCAN platform. We believe that these ongoing source and sensor programs will pave the way towards a comprehensive solution for actinic patterned mask inspection bridging the gap of actinic defect detection and identification on EUV reticles.
\end{abstract}

Keywords: Actinic patterned mask inspection, lensless scattering methods, EUV reticle, high frame-rate detector, EUV source.

\section{INTRODUCTION}

EUV lithography is expected to be inserted in high-volume manufacturing (HVM) for critical layers soon. Recent progress in various technological challenges ${ }^{1-3}$ have significantly increased the confidence level for manufacturing readiness. As the industry looks forward to the insertion of EUV into HVM, there are many challenges to be overcome. The success of EUV lithography now hinges on EUV mask infrastructure readiness. While, mask metrology tools, such as electron microscopy, are being adapted seamlessly for EUV reticles, ${ }^{4}$ the challenge in the mask ecosystem has always been the availability of actinic metrology tools. Defects embedded deep in the multilayer reflective coatings or on the mask substrate, are not accessible to a SEM or deep ultraviolet microscopy or defects on the patterned mask which are either elusive or falsely detected by these, make it neccessary for actinitic metrology solutions. ${ }^{5}$ In the last decade, several tool development programs were carried out for actinic blank inspection, ${ }^{6}$ aerial imaging ${ }^{7,8}$ and repair of the patterned masks, ${ }^{8}$ which are presently supporting EUV mask shops. A missing gap in the EUV mask ecosystem has been an actinic inspection tool for fast defect detection towards generating a full mask defect map. We address this void, with RESCAN ${ }^{9-12}$ (REflective mode EUV mask SCANnning lensless imaging tool), an EUV photomask inspection tool being developed at Paul Scherrer Institut in partnership with Nuflare Inc. Japan. In this paper, we review the lensless techniques behind RESCAN (Section 1), detail the currently operational tool at PSI (Section 2), provide experimental results on defect identification and aerial imaging retrieval from the prototype (Section 3) and finally conclude with an outlook for high-throughput stand-alone application (Section 4).

E-mail: rajeev.rajendran@psi.ch

Metrology, Inspection, and Process Control for Microlithography XXXI, edited by Martha I. Sanchez, Vladimir A. Ukraintsev Proc. of SPIE Vol. 10145, 101450N · C 2017 SPIE · CCC code: 0277-786X/17/\$18 · doi: 10.1117/12.2258379 


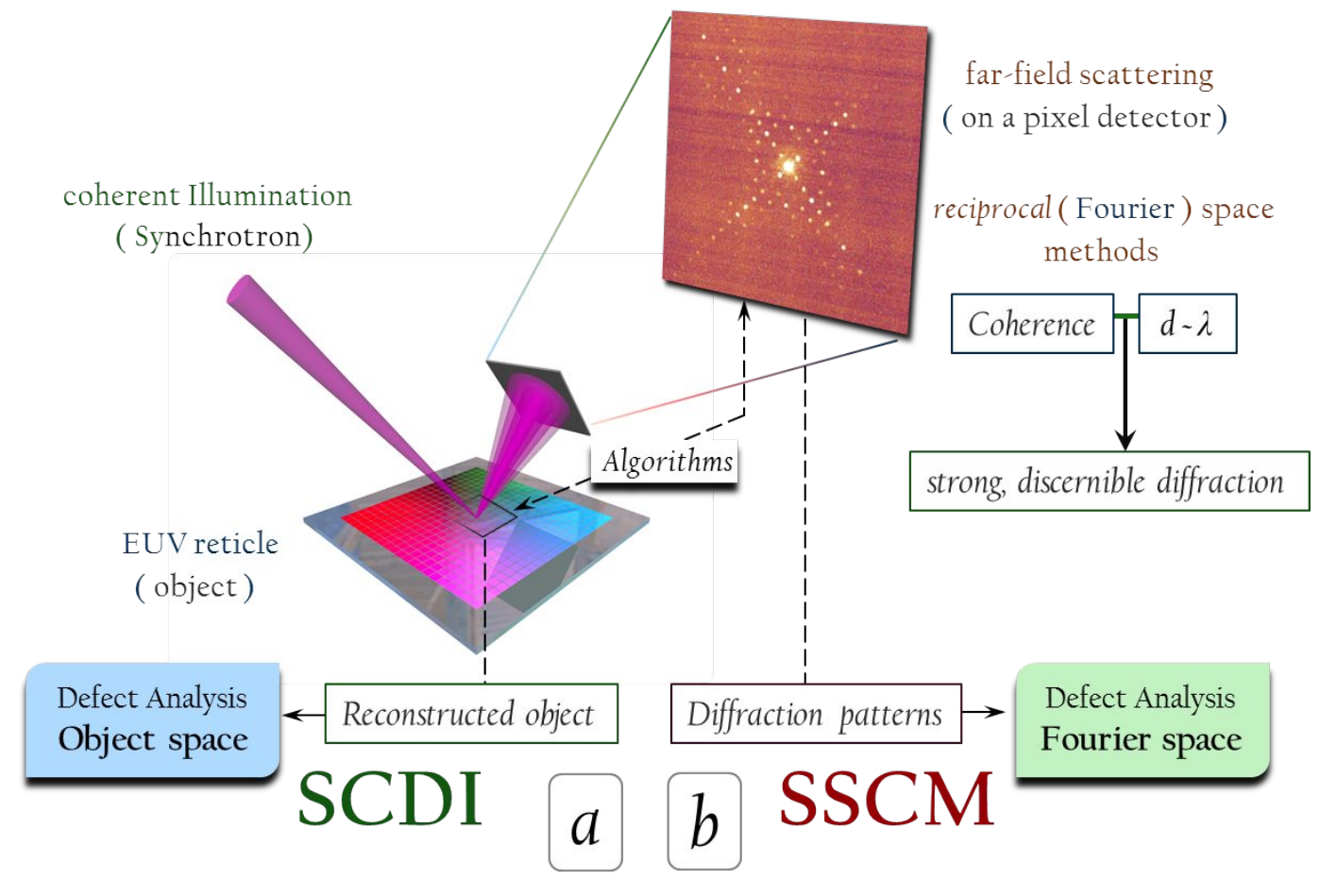

Figure 1. Coherent scattering techniques for defect analysis : The reticle is illuminated with coherent photons (from a synchrotron) and the far field diffraction image is recorded using a pixel detector. Here, $d$ is the length scale of the patterns on the photomask and $\lambda$, the illumination wavelength. The defect analysis is performed either (a) indirectly on the object space by reconstructing the object using phase retrieval algorithms from the measured diffraction images or (b) directly on the reciprocal space looking for Fourier signatures of the defect in the diffraction images. The former corresponds to the SCDI technique and the latter to SSCM as described in the text.

\section{COHERENT SCATTERING TECHNIQUES FOR INSPECTION AND IMAGING}

The RESCAN tool is based on coherent scattering of EUV photons from the patterned reticle. ${ }^{9,10}$ By illuminating a certain field of the photomask with coherent photons (typically from a synchrotron source), the far field scattering intensity is recorded on a pixel detector. The coherence of the photons supplemented by the fact that the scale length $d$ of the illuminated structures is of the same order $(d \sim \lambda)$ as the wavelength $\lambda$ of the light wave, leads to strong diffraction in the far-field, which is the reciprocal space of the illuminated object (see Figure 1). RESCAN infers the presence of the mask defects and generates their aerial images by measurement and analysis of these far-field diffraction images, avoiding the use of any kind of collection or imaging optics post illumination.

The recorded spectral intensities of spatial frequencies in the Fourier space on one hand allows (a) direct defect analysis in that space (Scanning Scattering Contrast Microsopy - SSCM ${ }^{9,11}$ ) or (b) using phase retrieval algorithms, enable lens-free recovery of the aerial image of the reticle fields (Scanning Coherent Diffractive Imaging - SCDI ${ }^{10,13}$ ). These Fourier space techniques have the advantage that they are not limited in resolution due to aberrations of optics and are pellicle-ready due to the long working distance. ${ }^{9}$

\section{Scanning Scattering Contrast Microscopy - Fast Actinic Defect Detection}

The method of SSCM relies on identifying the defect signal directly in the measured diffraction images. The recovery of the Fourier signature of the defect requires a reference measured diffraction image of either the defectfree field (i.e. die-to-die inspection) or simulated Fourier spectrum of the EUV aerial image of the design layout (i.e. die-to-database inspection). ${ }^{9}$ In either case, SSCM aims to generate a coarse defect map, whose resolution is limited only by the spot size of illumination. The technique is illustrated in Figure 2 and detailed in Ref[10]. $[\mathrm{h}]$ 


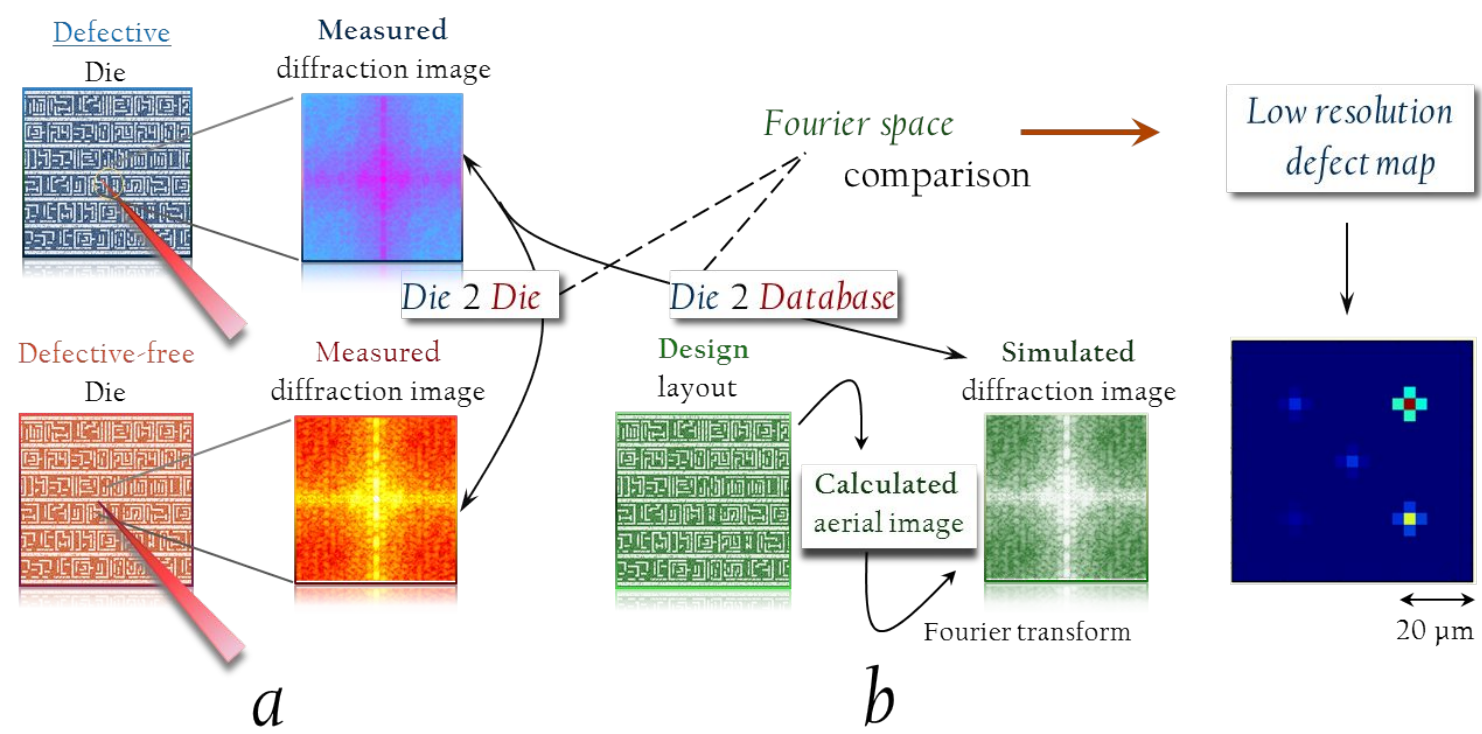

Figure 2. SSCM - Fourier space defect analysis : The principle of SSCM involve correlation of measured diffraction pattern from a certain field of the defective die (blue) either (a) with the measured diffraction pattern from a defect-free die ( orange - die to die comparison) or (b) with the calculated Fourier transform of the simulated aerial image of the design layout ( green - die to database comparison). In both cases by intensity comparison of Fourier spectrum, a coarse defect map is generated.

\section{Scanning Coherent Diffractive Imaging - Aerial Image Recovery}

Measured diffraction images can also be used to retrieve the aerial images of the object amplitude and phase, ${ }^{10}$ performing a defect analysis on the object space. This is an inverse problem where information only of the reciprocal space amplitudes (phase information is lost in measurment by the detector) is used to reconstruct the object. To retrieve the phase, SCDI analogous to schemes like ptychography, ${ }^{13}$ exploits data redundancy in measurements. ${ }^{10,12,13}$ Multiple sets of diffraction images from closely illuminated regions with substantial spatial overlap generates the required data redundancy (see Figure 3). Assuming a certain initial amplitude and phase for the unknown illuminating probe and the object, the phase retrieval algorithm projects the solutions between the object and reciprocal spaces and converges to a unique solution, subject to constraints that (a) the reconstructed object fields in overlapping zones must match and (b) the diffraction intensities in these solutions must agree with the measured ones.

The resolution of the reconstructed object is determined by the numerical aperture (NA) of the detector used and typically is much higher (tens of nanometers) than the spot size (several microns) of the probe. While SSCM gives a fast but coarse defect map, SCDI can localize these identified defects further in the aerial images with a large position accuracy of the order of few nanometers. The technique of SCDI is elaborated in Ref[11].

\section{RESCAN - PROTOTYPE TOOL}

Presently, the experiments for proof-of-the-principle of SSCM and SCDI is performed on a prototype tool installed at the Swiss Light Source (SLS) - a third generation synchrotron facility at PSI. The prototype tool consists of (a) a dedicated beamline - XIL-II-RESCAN, commissioned recently and (b) the RESCAN end station (see Figure 4). The photon source for the tool is an undulator providing spatially-coherent EUV light with a bandwidth of $4 \%(E / \Delta E \sim 20)$. To obtain quality diffraction images, the temporal coherence is increased, i.e. the bandwidth is reduced, using a grating monochromator to a monochromaticity of $E / \Delta E \sim 1600$ achieving a coherence length of about $30 \mu \mathrm{m}$.

The end station (see Figure $4 \mathrm{c}, \mathrm{d}$ ), coupled to the RESCAN beamline, consists mainly of focusing optics for illumination, the sample handler and EUV pixel detector for capturing the far field scattering. To ensure 

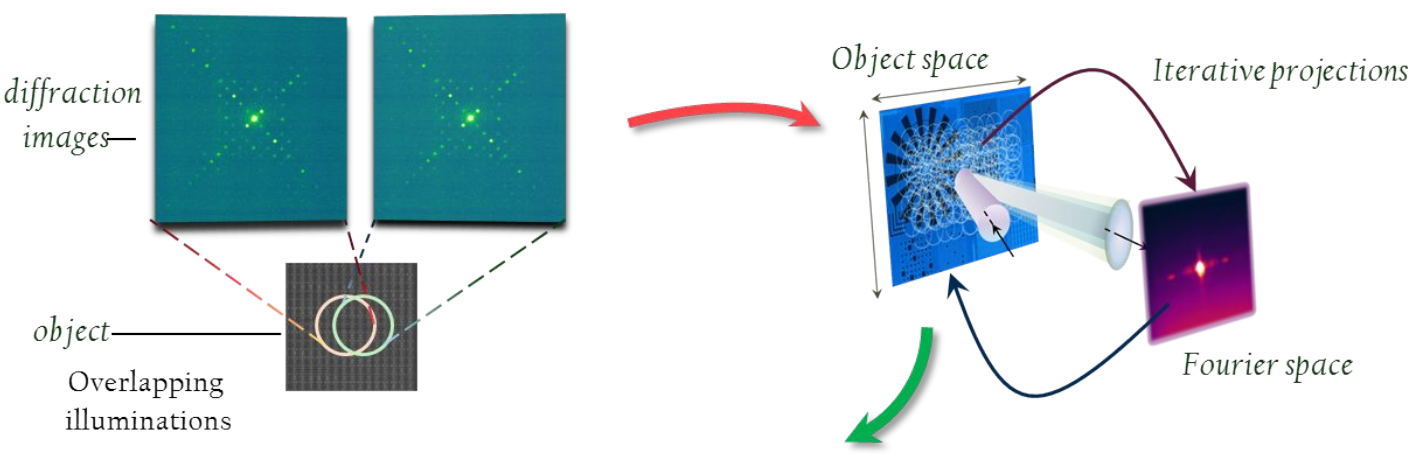

C High resolution Aerial images

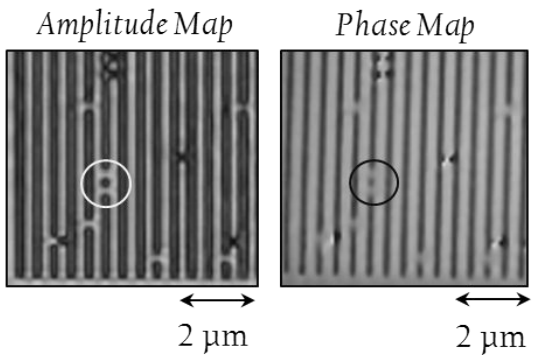

Figure 3. SCDI - Aerial image recovery and object space defect analysis : (a) The measurement for SCDI involve collecting diffraction patterns from adjacent illumination zones ensuring substantial overlap between them, thus ensuring data redundancy in the measurement procedure. (b)The iterative algorithm projects solutions between the object and reciprocal spaces subject to constraints (see text). (c) The aerial images of both the amplitude and phase of the object is simultaneously recovered. The circled regions shows an example of defect detection and its amplitude and phase characterization in the aerial image.

vibration-free measurements, the sample handling stage and the optics are coupled into the same breadboard. The light is focused onto the sample with a condenser mirror at an illumination angle of $6^{\circ}$ to a spot size of several microns. A sliding adaption allowed moving the detector to/away from the sample there by allowing a means to change the NA of the detector which determines the spatial resolution on the sample.

\section{EXPERIMENTAL RESULTS}

\section{Defect detection with SSCM}

To demonstrate reciprocal space analysis of defects with SSCM, we have considered two defective samples. Sample 1 consists of $100 \mathrm{~nm}$ half-pitch (on-mask dimensions) gratings with CD error of $10 \mathrm{~nm}$ across the entire field in one of the lines. Sample 2 on the other hand has a localized defect, again a CD error of $10 \mathrm{~nm}$ but spanning only $5 \mu \mathrm{m}$, on a $100 \mathrm{~nm}$ half-pitch grating. This localized patterned defect is positioned at the center of the sample field to correlate against any false non patterned particle defects due to contamination. In both cases, the basic field had a dimension of $40 \mu \mathrm{m} \times 40 \mu \mathrm{m}$. The probe size was determined by sampling it using an isolated $2 \mu \mathrm{m}$ contact hole embedded in an absorbing $\mathrm{Ni}$ coated multilayer mirror. By capturing the reflection from the sampling contact hole on the pixel detector, the intensity distribution of the probe at the sample plane was recovered. ${ }^{12}$ The generated defect maps using die to die comparison of the Fourier spectral intensities for both the samples are shown in Figure 5. 


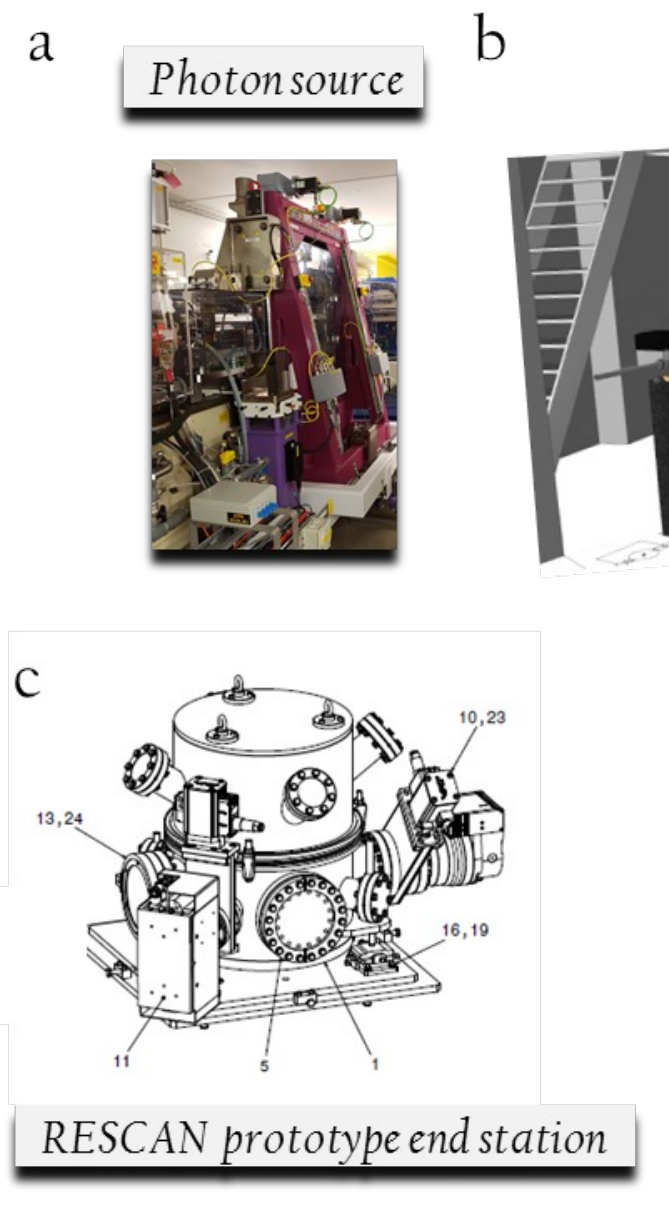

\section{Beamlinelayout

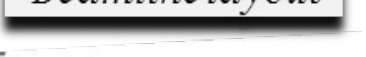

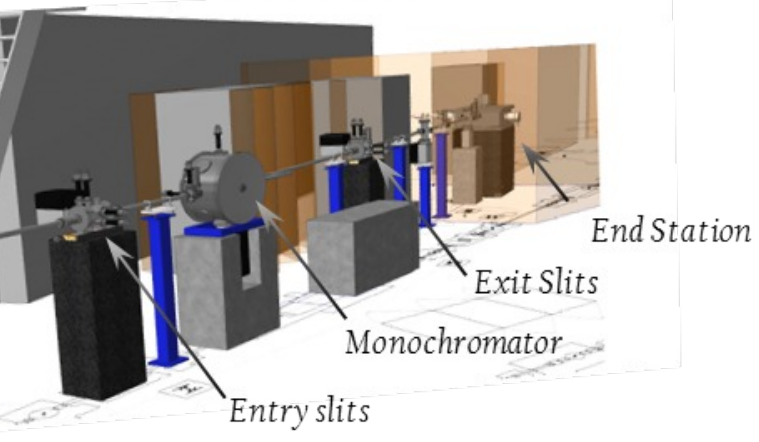

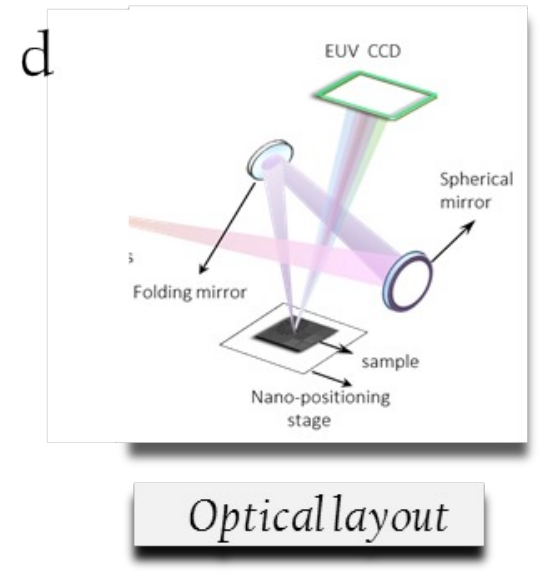

Figure 4. RESCAN prototype tool : The prototype tool operational at Swiss Light Source is powered by an (a) undulator source and the beam is conveyed to the end station through a (b) newly commissioned beamline with an integrated monochromator. (c) The RESCAN end station and (d) the optical lay out.

\section{Defect localization with SCDI}

To obtain high resolution images of the patterned masks, aerial image of the sample was recovered using SCDI (see Figure $6,7,8$ ). The sample in this case consisted of a multilayer coated Si wafer substrate with HSQ absorber patterns. Like for SSCM samples each field spanning $90 \mu m \times 90 \mu m$ was repeated periodically over a zone of $2 \mathrm{~mm} \times 2 \mathrm{~mm}$. The large spectrum of features on the sample (see Figure 7) catered to a range of feature sizes. The images recovered through SCDI compare very well with those obtained using SEM. The defects on the gratings as seen by the SEM are visible in the RESCAN images too.

\section{OUTLOOK}

While the results from the prototype tool is promising, in order to migrate the tool from a research platform demonstrating the capability of coherent scattering techniques in defect detection to a standardized industryready platform for actinic mask inspection, few issues beyond the techniques themselves need to be addressed. The industrial acceptance of defect inspection tools of photomasks is measured by three yardsticks - throughput, sensitivity and resolution in detecting defects. We plan for major upgrades to reach industrial target specifications. 
a

+10 nm full field CD error

$100 \mathrm{~nm}$ hp grating

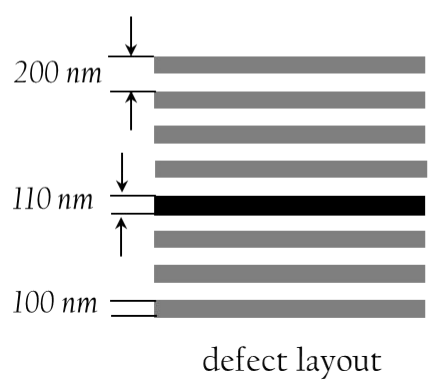

b

+10 nm x 5000 nm localized CD error

$100 \mathrm{~nm}$ hp grating

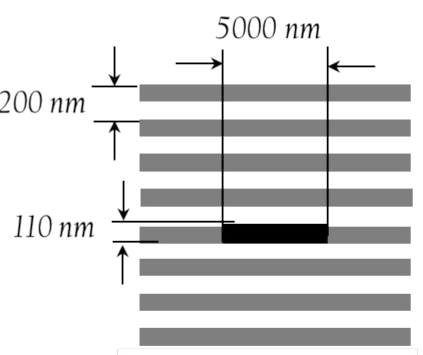

defect layout

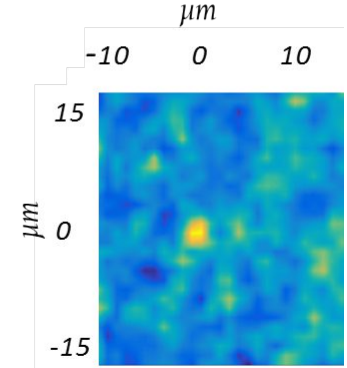

defect map

Figure 5. SSCM - defect map results : The defect map obtained using die to die comparison of diffraction images from $100 \mathrm{~nm}$ hp gratings are shown (a) for a CD error of $+10 \mathrm{~nm}$ on mask in one of the lines across the entire field and (b) for a localized defect of $+10 \mathrm{~nm} \times 5000 \mathrm{~nm}$ on mask. The defect lay out shown are not to scale but is projected for visualization only. The pupil conditions and hence the illuminating spot sizes for (a) differed from (b) as reflected in the width of the defect signal in the defect map.

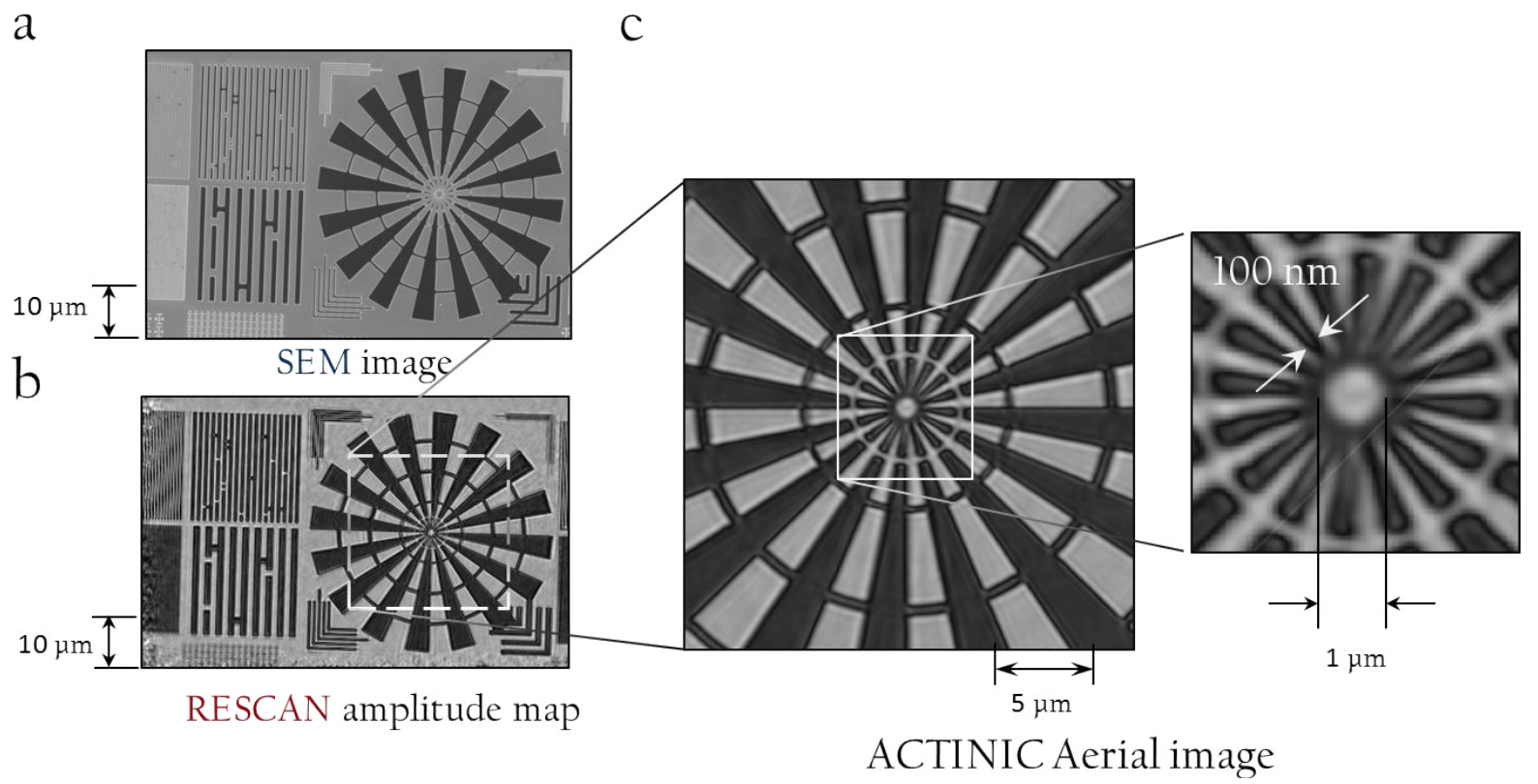

Figure 6. SCDI - RESCAN images vs SEM images : Images showing the sample photomask used for SCDI. (a) SEM (b) actinic aerial image (amplitude map) retrieved using SCDI from RESCAN. (c) A zoomed image of (b) clearly showing well resolved $100 \mathrm{~nm}$ spokes of the Siemens star.

\section{Throughput}

The targeted scan time specified for full mask inspection is 7 hours. With an illumination spot of $30 \mu m$ and assuming illumination overlap of $50 \%$ between consecutive illumination spots, this corresponds to $\sim 5 \times 10^{7}$ images translating to an image acquisition rate of $1.8 \mathrm{kHz}$. The presently available EUV CCD detectors have frame rates of a few $\mathrm{Hz}$. A high frame rate EUV detector is the first and foremost requirement for high through- 


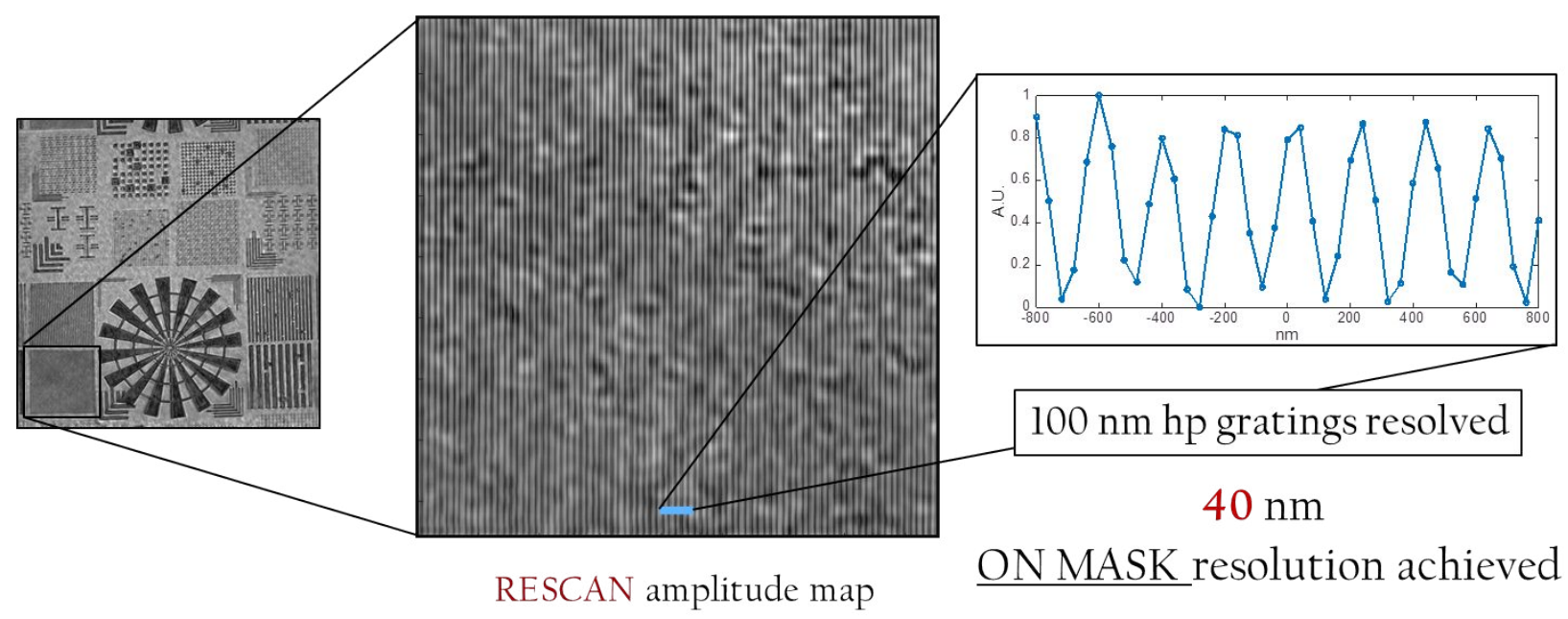

Figure 7. SCDI - on mask resolution : The gratings from the imaged field in (a) was used to evaluate the resolution of the system empirically. The plot in (b) is a line cut ( marked blue ) across the grating. The resolution was estimated by quantifying the spatial extend of an edge between $90 \%$ and $10 \%$ of the line intensity in this plot and was found to be $\sim 40 \mathrm{~nm}$ on mask. The designed detector NA limited resolution is $32 \mathrm{~nm}$, indicating good system performance both in terms of measurements and reconstruction.

a

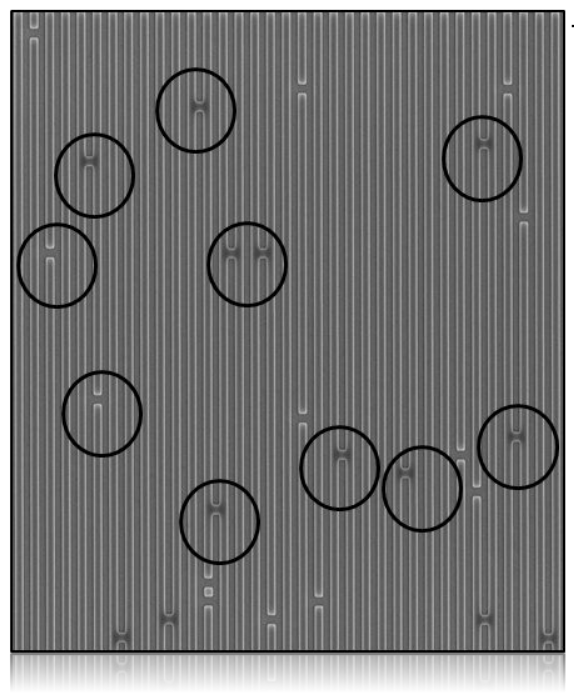

$\mathrm{b}$

\begin{tabular}{l}
$\sqrt{2} \mu \mathrm{m}$ \\
$250 \mathrm{~nm}$ hp \\
gratings \\
with \\
pre-patterened \\
defects \\
ON MASK \\
\hline
\end{tabular}

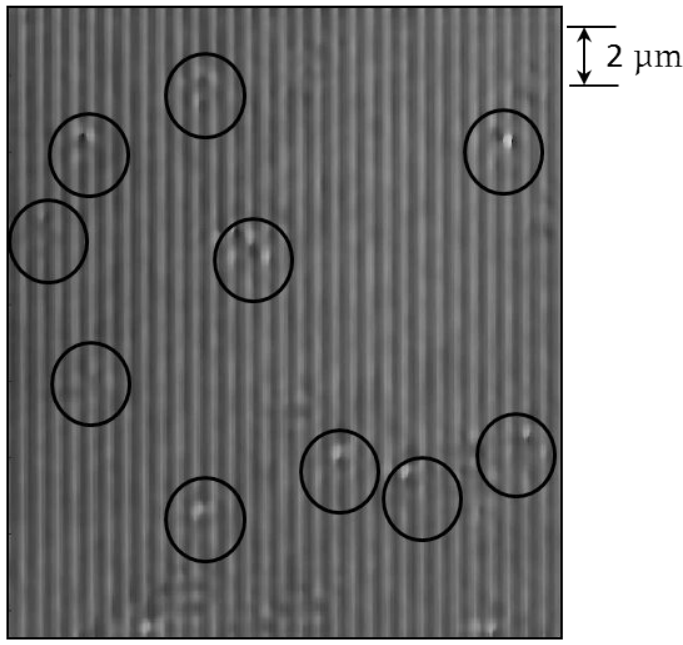

RESCAN phase map

Figure 8. SCDI - defect localization : The phase map recovered in the reconstruction of the aerial images could also be used for identifying and localizing the defects in an actinic map. Here (a) corresponds to SEM image of a field of 200 nm half pitch grating embedded with multiple patterned defects. (b) This is a phase map of the aerial image of the same field. A large proportion of the patterned defects could be detected and well localized by RESCAN generated phase map.

put inspection. A large frame rate of $2 \mathrm{kHz}$ also would mean that the exposure time per illumination spot is restricted to less than $500 \mu \mathrm{s}$. To register significant number of scattered photons from small defects on the detector in such short duration with acceptable signal-to-noise ratio, a coherent source with high brightness as well as sufficient flux is equally a crucial component. In summary, for a high throughput inspection, a high frame 
a

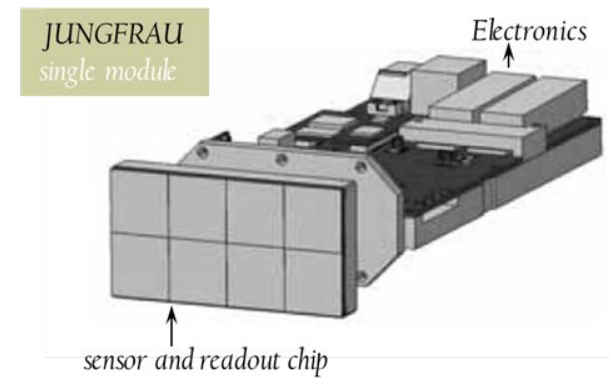

$\mathrm{b}$

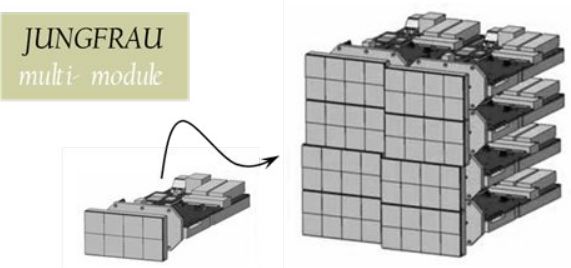

C

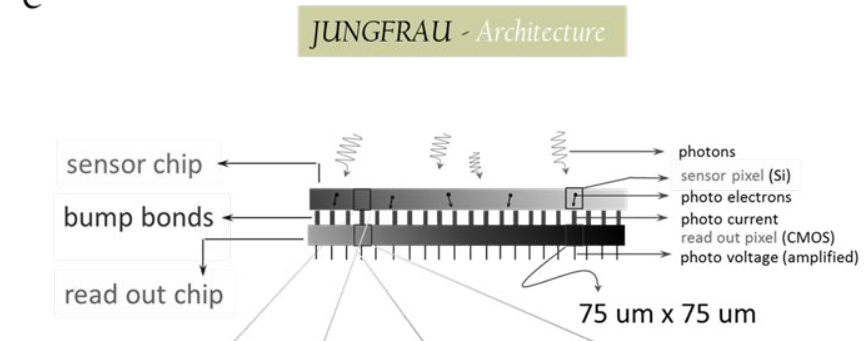

$75 \mathrm{um} \times 75 \mathrm{um}$

Read Out Pixel

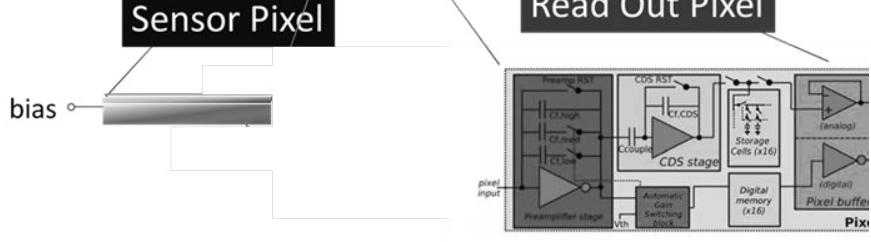

Figure 9. JUNGFRAU - mechanical layout and architecture : (a) JUNGFRAU single module showing the detection system in the foreground and the data processing electronics. (b) The simple layout of the modules allows to scale the detector size easily. (c)The detailed architecture of the Hybrid detection scheme. The sensor and the readout chip are independently devised and coupled at every pixel by wire bonding performed in house. The JUNGFRAU individual pixel are $75 \mu m \times 75 \mu m$.

rate detector and a bright coherent source are needed.

\section{Sensitivity}

The technique of SSCM relies on distinguishing a significantly lower count of photons due to defects in the Fourier plane compared to those from the main diffraction peaks. The low intensity of the defect signal is firstly due to the relatively smaller area of the defects $(\sim 10 \mathrm{~nm})$ with respect to the focal spot of illumination $(\sim 10 \mu m)$, and secondly due to the nature of Fourier signature of small defects - smaller the defects farther they spread in the diffraction image and consequently lower the signal strength per pixel. In the die-to-die comparison for example, its important to simultaneously capture the intense peaks of the design patterns (without saturation) and the very low photon counts from the defect signal in the same image. This translates to a dynamic range of $\sim 10^{5}$ or above. Currently available commercial CCDs for EUV have well potentials that provide a maximum dynamic range of $10^{4} \mathrm{EUV}$ photons.

\section{$\underline{\text { Resolution }}$}

The aerial image recovery using SCDI is limited in resolution by the NA of the detector, similar to the conventional imaging. While the easiest way to scale the NA is to bring the sensor closer to the sample, geometric restrictions of $6^{\circ}$ illumination limits the resolution.

\section{A high frame rate high dynamic range EUV sensor - the JUNGFRAU}

Presently, there are no commercial detector solution which caters to all the specifications mentioned above. Among the detector platforms under development, we adopted the hybrid active pixel CMOS detector which is developed at PSI. The JUNGFRAU (adJUstiNg Gain detector FoR the Aramis User station) detector is already 


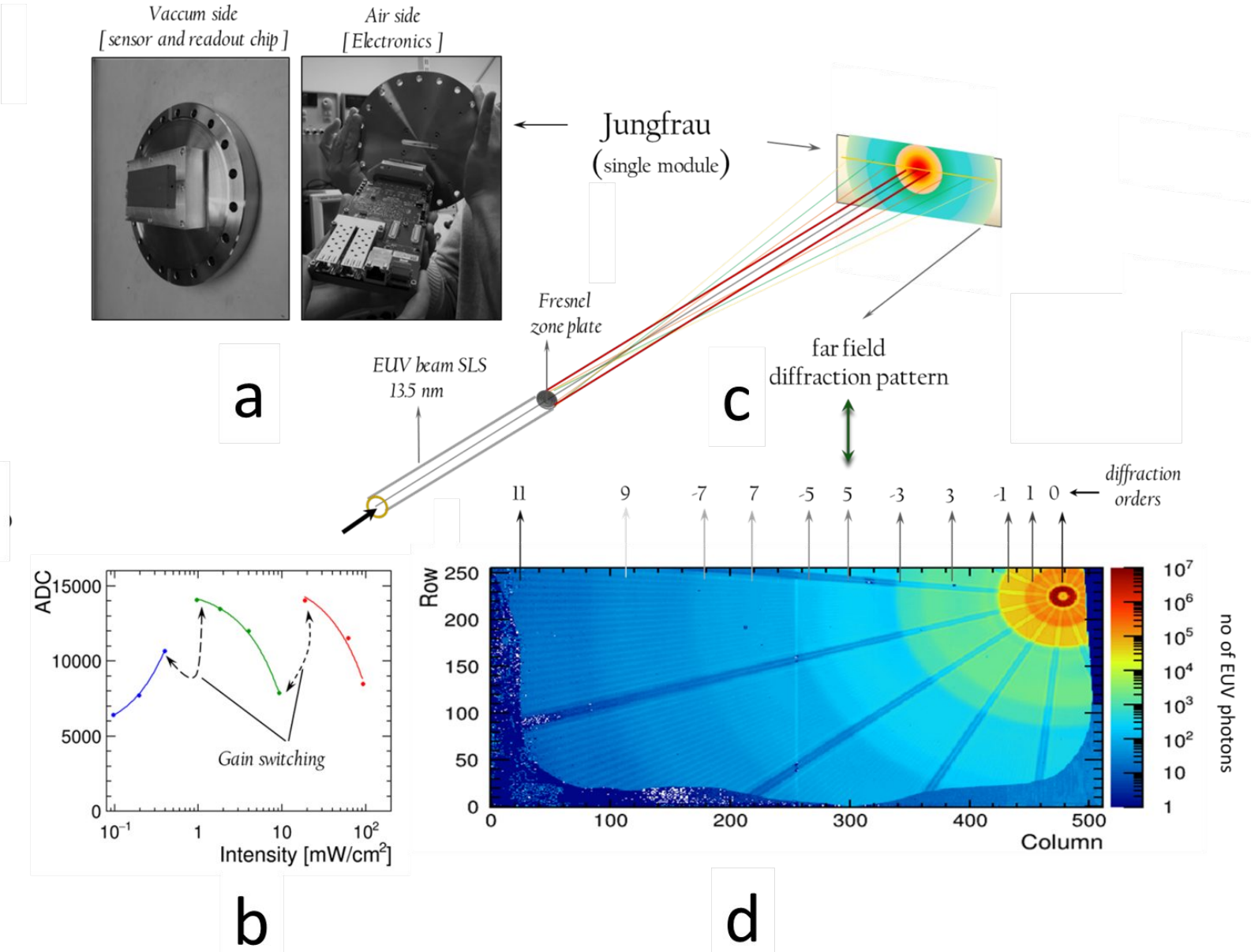

Figure 10. JUNGFRAU - EUV performance : To benchmark the performance of JUNGFRAU for $13.5 \mathrm{~nm}$ photons, a vacuum compatible single module was specially designed. (a) The sensor is housed in the vacuum side while the electrons is in the air side. (b) The gain switching of the detector was verified for EUV photons. The linearity of the system was also checked. (c) The experimental lay out to measure the dynamic range of JUNGFRAU for EUV radiation. A Fresnel zone plate in the path of coherent photons from the synchrotron gives diffraction in the far field with its orders spanning a large range of intensities. JUNGFRAU was used to measure these orders. (d) The measurements provide clear images with $11^{\text {th }}$ order well resolved above the noise level, despite their weak signal strength by 7 orders of magnitude. With its dynamic gain switching JUNGFRAU offers a dynamic range $>10^{6}$ for EUV photons.

a relatively mature technology, originally designed for free electon laser (FEL) applications. ${ }^{14-16}$ It will reach the requirements for EUV mask inspection after further developments, in particular, in EUV sensitivity improvement (see Figure 9). JUNGFRAU is a charge integrating detector that can register images with large photon flux in a short temporal span as is usual with diffraction images from coherent scattering of intense ultrashort photon pulses from FELs. It is a silicon based hybrid detector solution. It has two functional layers - a sensor layer and a read out chip below. Each pixel $(75 \mu m \times 75 \mu m)$ on the sensor is associated with a corresponding read out pixel(see Figure 9c). The two layers are coupled by pixel-to-pixel wire bonding. This allows massive parallelization in data transfer and consequently a high frame rate. A large dynamic range of photons within every pixel is realized by allowing the same ADC range to read a larger range of photo electrons by automatically adjusting the gain above certain thresholds of photoelectron current. By using a set of three capacitors in every readout pixel, the photoelectrons are distributed among them according to the thresholding values. The gain bits of the respective gain stages are then registered by the 'fill' or 'no fill' status of the respective capacitors. 


\section{COSAMI}

\section{Compact EUV Source for Actinic Mask Inspection}

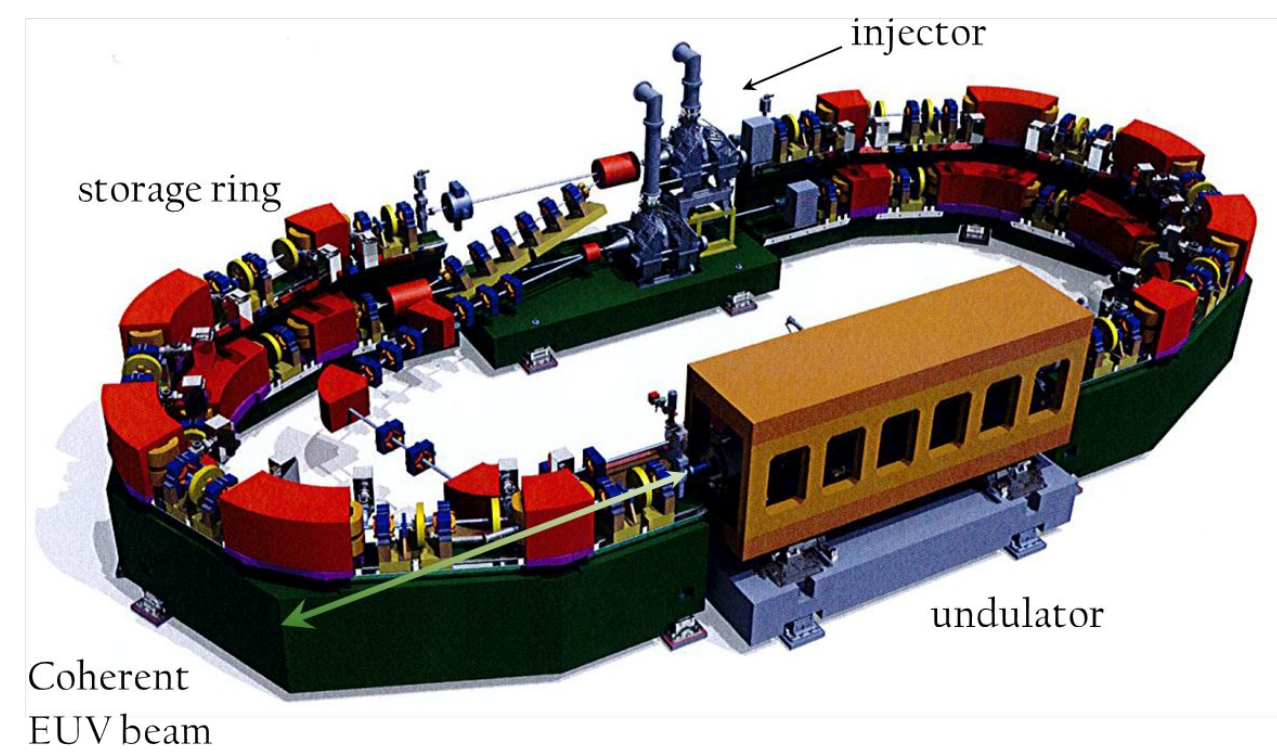

Figure 11. Towards stand alone operation-COSAMI : (a) The in-house coherent EUV source, conceptualized and designed at PSI and optimized for EUV photons. COSAMI operates in a top-up mode providing $100 \mathrm{~mW}$ of power with a relatively small footprint.

The final output is then read out as combination of ADC and the gain bits.

We have measured a dynamic range of about $10^{6}$ at EUV wavelength. These measurements involved recording the diffraction pattern of Fresnel zone plate illuminated by coherent EUV beam (see Figure 10). In addition, the electronic, system and mechanical design of the detector allows tiling of multiple modules(see Figure 9b). The sensor in its present version is optimized for soft x-rays with thick front end window and hence provides poorquantum efficiency for EUV. A new EUV-optimized sensor chip is under development to increase the EUV detection efficiency substantially. With the massive parallelization of the readout architecture, JUNGFRAU offers a frame rate of $2 \mathrm{kHz}$ (scalable to $4 \mathrm{kHz}$ with firmware changes).

\section{A compact bright source for coherent EUV photons - the COSAMI}

RESCAN needs a coherent EUV source with high brighness. Since the metrology method is based on coherent scattering its brightness requirement is significantly higher compared to lens-based imaging or inspection methods. Whereas high-harmonic generation sources ${ }^{17}$ provide coherent EUV light, their ultimately achievable flux is not sufficient for high-throughput inspection. Presently, high-brigthness and flux sources are only available in large-scale facilities and RESCAN presently is docked to one such beamline. From a long term point of view of integration and sustainability within a mask shop ecosystem, the tool needs to migrate to a stand-alone platform. Therefore, we have envisaged and conceptualize a compact source for bright coherent EUV photons based on proven and well-established accelerator-based technologies. In collaboration with the accelerator and source development division at PSI, we present here COSAMI ${ }^{18}$ - a COmpact Source for Actinic Mask Inspection. COSAMI has a small foot print of about $5 \times 12 \mathrm{~m}^{2}$ and is optimized specifically for EUV metrology applications. (see Figure 11). It accomodates a linear accelerator (LINAC), a booster ring for further acceleration and an electron storage ring in a concentric design and a single undulator, providing flux of about $100 \mathrm{~mW}$ with $0.5 \%$ bandwidth and brilliance of $1.8 \times 10^{18}$ photons $/ \mathrm{s} / \mathrm{mm}^{2} / \mathrm{mrad}^{2} / 0.1 \% \mathrm{BW}$. The system delivers a $10^{-3}$ intensity 
stability, which is realized by frequent injection from the booster ring to the storage ring ensuring steady photon flux. The technology is currently in conceptual and technical design phase.

\section{CONCLUSIONS}

In summary, the current RESCAN tool providea proof-of-principle experiments for both SSCM and SCDI. We plan for significant upgrades in all aspects of the tool and integrate co-developed technologies, which will enable us to upscale the performance of the tool. The essential techniques and the necessary technological components for a stand alone and high throughput tool is either feasible or availabe today. We have a clear path towards the realization of a tool that could meet the targeted specifications of the mask shops.

\section{ACKNOWLEDGMENTS}

We would like to make special thanks to all the members of the XIL and SIM beamlines at Swiss Light Source for the support with the smooth operation of the beamline and endstation. A special word of mention to all the members of source team at PSI for the design of COSAMI, including Masamitsu Aiba, Ryan Mitchell Bergmann, Theo Bieri, Paolo Craievich, Michael Ehrlichman, Terence Garvey, Christopher Gough Philippe Lerch, Andreas Mller, Marco Negrazus Leonid Rivkin, Colette Rosenberg, Lothar Schulz, Lukas Stingelin, Andreas Streun, Vjeran Vrankovic, Albin Wrulich, Adriano Zandonella Callegher, Riccardo Zennaro.

\section{REFERENCES}

[1] Mizoguchi, H., Nakarai, H., Abe, T., Nowak, K. M., Kawasuji, Y., Tanaka, H., Watanabe, Y., Hori, T., Kodama, T., Shiraishi, Y., Yanagida, T., Yamada, T., Yamazaki, T., Okazaki, S., and Saitou, T., "Performance of new high-power hvm lpp-euv source," Proc. SPIE 9776, 97760J-97760J-8 (2016).

[2] Yamazaki, T., Mizoguchi, H., Nakarai, H., Abe, T., Kawasuji, Y., Okamoto, T., Tanaka, H., Watanabe, Y., Hori, T., Kodama, T., Shiraishi, Y., Okazaki, S., and Saitou, T., "Key components technology update of 100w hvm euv source," Proc. SPIE 9422, 94222P-94222P-7 (2015).

[3] Lio, A., "Euv resists: What's next?," Proc. SPIE 9776, 97760V-97760V-14 (2016).

[4] Hirano, R., Iida, S., Amano, T., Watanabe, H., Hatakeyama, M., Murakami, T., Yoshikawa, S., Suematsu, K., and Terao, K., "Patterned mask inspection technology with projection electron microscope (pem) technique for $11 \mathrm{~nm}$ half-pitch (hp) generation euv masks," Proc. SPIE 9658, 96580L-96580L-8 (2015).

[5] Mochi, I., Goldberg, K. A., La Fontaine, B., Tchikoulaeva, A., and Holfeld, C., "Actinic imaging of native and programmed defects on a full-field mask," Proc. SPIE 7636, 76361A-76361A-9 (2010).

[6] Suzuki, T., Miyai, H., Takehisa, K., Kusunose, H., Watanabe, H., and Mori, I., "Detection capability of actinic blank inspection tool," Proc. SPIE 9658, 96580O-96580O-6 (2015).

[7] Goldberg, K. A., Mochi, I., Benk, M. P., Lin, C., Allezy, A., Dickinson, M., Cork, C. W., Macdougall, J. B., Anderson, E. H., Chao, W., Salmassi, F., Gullikson, E. M., Zehm, D., Vytla, V., Cork, W., DePonte, J., Picchi, G., Pekedis, A., Katayanagi, T., Jones, M. S., Martin, E., Naulleau, P. P., and Rekawa, S. B., "The sematech high-na actinic reticle review project (sharp) euv mask-imaging microscope," Proc. SPIE 8880, 88800T-88800T-9 (2013).

[8] Hellweg, D., Perlitz, S., Magnusson, K., Capelli, R., Koch, M., and Malloy, M., "Actinic review of euv masks: performance data and status of the aims euv system," Proc. SPIE 9776, 97761A-97761A-8 (2016).

[9] Mohacsi, I., Helfenstein, P., Rajendran, R., and Ekinci, Y., "Scanning scattering contrast microscopy for actinic euv mask inspection," Proc. SPIE 9778, 97781O-97781O-11 (2016).

[10] Helfenstein, P., Mohacsi, I., Rajeev, R., and Ekinci, Y., "Scanning coherent diffractive imaging methods for actinic extreme ultraviolet mask metrology," Journal of Micro/Nanolithography, MEMS, and MOEMS 15(3), 034006 (2016).

[11] Ekinci, Y., Helfenstein, P., Rajeev, R., Mochi, I., Mohacsi, I., Gobrecht, J., and Yoshitake, S., "Scanning coherent scattering methods for actinic euv mask inspection," Proc. SPIE 9985, 99851P-99851P-9 (2016).

[12] Mochi, I., Helfenstein, P., , Mohacsi, I., Rajeev, R., Yoshitake, S., and Ekinci, Y., "Rescan: An actinic lensless microscope for defect inspection of euv reticles," Proc. SPIE 10143, - (2017). 
[13] Thibault, P., Dierolf, M., Menzel, A., Bunk, O., David, C., and Pfeiffer, F., "High-resolution scanning x-ray diffraction microscopy," Science 321(5887), 379-382 (2008).

[14] Smith, J. H., Mozzanica, A., and Schmitt, B., "Jungfrau a dynamic gain switching detector for swissfel," Technical Design Report (2015).

[15] Rajeev, R., Redford, S., Mochi, I., Helfenstein, P., Mohacsi, I., A.Mozzanica, Schmitt, B., and Ekinci, Y., "A high-frame rate pixel detector with extended dynamic range for euv imaging and mask metrology," EUVL Symposium 2016, Hiroshima (2016).

[16] Redford, S., Bergamaschi, A., Brckner, M., Cartier, S., Dinapoli, R., Ekinci, Y., Frjdh, E., Greiffenberg, D., Mayilyan, D., Mezza, D., Mozzanica, A., Rajeev, R., Ramilli, M., Ruder, C., Schdler, L., Schmitt, B., Shi, X., Thattil, D., Tinti, G., and Zhang, J., "Calibration status and plans for the charge integrating jungfrau pixel detector for swissfel," Journal of Instrumentation 11(11), C11013 (2016).

[17] Rudawski, P., Heyl, C. M., Brizuela, F., Schwenke, J., Persson, A., Mansten, E., Rakowski, R., Rading, L., Campi, F., Kim, B., Johnsson, P., and LHuillier, A., "A high-flux high-order harmonic source," Review of Scientific Instruments 84(7), 073103 (2013).

[18] L.Rivkin, "Light sources based on storage rings," Introduction to Accelerator Physics, Budapest (2015). 